\title{
ON THE HONEYCOMB CONJECTURE FOR ROBIN LAPLACIAN EIGENVALUES
}

\author{
DORIN BUCUR, ILARIA FRAGALÀ
}

\begin{abstract}
We prove that the optimal cluster problem for the sum of the first Robin eigenvalue of the Laplacian, in the limit of a large number of convex cells, is asymptotically solved by (the Cheeger sets of) the honeycomb of regular hexagons. The same result is established for the Robin torsional rigidity.
\end{abstract}

\section{Introduction AND STATEMENT OF the RESUlts}

Given an open bounded Lipschitz domain $\Omega$ in $\mathbb{R}^{2}$ and a real parameter $\beta \neq 0$, we denote by $\lambda_{1}(\Omega, \beta)$ and $\tau(\Omega, \beta)$ the first Robin eigenvalue of the Laplacian in $\Omega$ and the Robin torsional rigidity of $\Omega$ with coefficient $\beta$. They are defined as

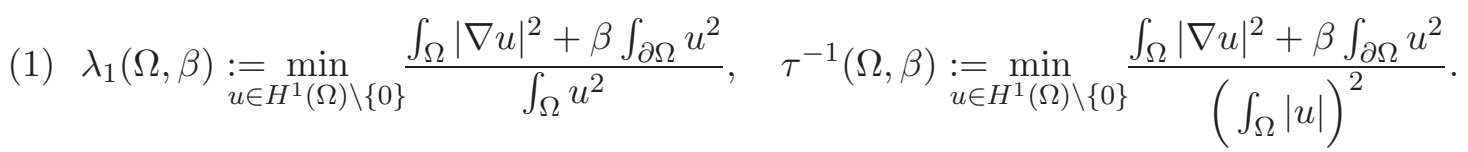

For the eigenvalue problem, the corresponding Euler-Lagrange equation is given by

$$
\begin{cases}-\Delta u=\lambda_{1}(\Omega, \beta) u & \text { in } \Omega \\ \frac{\partial u}{\partial \nu}+\beta u=0 & \text { on } \partial \Omega .\end{cases}
$$

For the torsional rigidity, the Euler-Lagrange equation requires more attention (see for instance [2]), specifically in the case $\beta<0$. For positive $\beta$, the minimizer solves

$$
\begin{cases}-\Delta u=1 & \text { in } \Omega \\ \frac{\partial u}{\partial \nu}+\beta u=0 & \text { on } \partial \Omega\end{cases}
$$

while for negative $\beta$, the Euler-Lagrange equation may involve a free boundary problem. It is not the purpose of the present paper to discuss this issue, as we focus only on the energy values defined in (11). Without any attempt of completeness, we refer to [9, 10, 11] for some recent papers in shape optimization involving free boundaries with Robin conditions.

While there is a wide literature about optimal partitions for the first Dirichlet Laplacian eigenvalue (see for instance [3, 4, 6, 15, 16, 23, 24, 25, 31]), to the best of our knowledge the study of the same kind of problem for the first Robin Laplacian eigenvalue is a completely unexplored field.

Object of this paper are the optimization problems

$$
r_{k}(\Omega, \beta)=\left\{\begin{array}{l}
\inf \left\{\sum_{i=1}^{k} \lambda\left(E_{i}, \beta\right):\left\{E_{i}\right\} \in \mathcal{C}_{k}(\Omega)\right\} \text { if } \beta>0 \\
\sup \left\{\sum_{i=1}^{k} \lambda\left(E_{i}, \beta\right):\left\{E_{i}\right\} \in \mathcal{C}_{k}(\Omega)\right\} \text { if } \beta<0
\end{array}\right.
$$

2010 Mathematics Subject Classification. 52C20, 51M16, 65N25, 49 Q10.

Key words and phrases. Optimal partitions, honeycomb, Robin Laplacian eigenvalues, $\alpha$-Cheeger constant. 
where $\mathcal{C}_{k}(\Omega)$ denotes the class of convex $k$-clusters $\Omega \subset \mathbb{R}^{2}$, meant as families of $k$ convex bodies contained into $\Omega$ and having mutually disjoint interiors, and, for any $\beta \in \mathbb{R} \backslash\{0\}$, $\lambda(\Omega, \beta)$ may be either $\lambda_{1}(\Omega, \beta)$ or $\tau^{-1}(\Omega, \beta)$.

We are interested in particular in the asymptotic behaviour of $r_{k}(\Omega, \beta)$ in the limit as $k \rightarrow+\infty$. Our main motivation is a conjecture due to Caffarelli and Lin [12] which predicts that, for the analogous problems in which $\lambda(\Omega, \beta)$ is replaced by the first Dirichlet Laplacian eigenvalue, an optimal configuration is asymptotically given by a packing of regular hexagons, similarly to the case of perimeter minimizing partitions settled by Hales in the celebrated paper [22] (see also [14] for a quantitative formulation).

Very recently, in [8] this conjecture has been proved to hold if one takes the Cheeger constant in place of the Dirichlet eigenvalue, and the cells of the partitions are a priori assumed to be convex. Recall that the Cheeger constant of $\Omega$ (about which a detailed account can be found for instance in [28, 29]) is defined by

$$
h(\Omega):=\inf \left\{\frac{\operatorname{Per}\left(E, \mathbb{R}^{2}\right)}{|E|}: E \text { measurable }, E \subseteq \Omega\right\},
$$

where $\operatorname{Per}\left(E, \mathbb{R}^{2}\right)$ denotes the perimeter of $E$ in the sense of De Giorgi.

Clearly, the fact that the notion of Cheeger constant is purely geometrical makes the analysis of optimal partitions, started by Caroccia in [13, much more manageable with respect to the case of eigenvalues. Nevertheless, the approach proposed in [8] does not rely specifically on the definition of $h(\Omega)$, but rather on the validity of a discrete Faber-Krahn inequality (which in case of the Cheeger constant has been proved in [7]) and on some other geometric properties, like the monotonicity upon inclusions of sets and a scaling behaviour; and in fact, the proof given in 8] adapts also to other shape functionals for which a polygonal version of Faber-Krahn inequality is available, such as for instance a power of perimeter or the logarithmic capacity (see [32]).

Now, a polygonal Faber-Krahn inequality for eigenvalues of the Laplacian is a longstanding conjecture by Pólya, for which a proof is still missing (see for instance [26]); thus the conjecture by Caffarelli-Lin remains open.

Under Robin boundary conditions, neither for the first Laplacian eigenvalue nor for torsional rigidity, polygonal isoperimetric inequalites are known (even for triangles); furthermore, neither $\lambda_{1}(\Omega, \beta)$ nor $\tau(\Omega, \beta)$ behave monotonically under inclusions. At this point, our results about the honeycomb conjecture for such Robin functionals should sound somewhat unexpected. We stress that we keep the assumption that the cells of the partitions are convex. In case of the first Robin eigenvalue, we prove:

Theorem 1. Let $r_{k}(\Omega, \beta)$ be defined by (2), with $\lambda(\Omega, \beta):=\lambda_{1}(\Omega, \beta)$. Then there holds

$$
\lim _{k \rightarrow+\infty} \frac{|\Omega|^{1 / 2}}{k^{3 / 2}} r_{k}(\Omega, \beta)=\beta h(H),
$$

where $h(H)$ denotes the Cheeger constant of the unit area regular hexagon.

Theorem [1 is obtained as a consequence of the analogous result proved in [8] for the Cheeger constant, combined with a tight control of the Robin eigenvalues in terms of the quotient perimeter over area ( $c f$. Proposition 9 below) when the number of cells is increasing. The idea is that, when there is a great amount of cells $E_{i}$, thanks to the non-scale invariance of the Robin eigenvalue, for a sufficiently large number of indices $i$, the value of $\lambda_{1}\left(E_{i}, \beta\right)$ turns out to be comparable to $\beta\left|\partial E_{i}\right| /\left|E_{i}\right|$, so that the partition behaves like a Cheeger one. From a technical point of view, the key point is to prove that 
this comparison can be made uniform, except for a negligible number of cells, which do not affect the asymptotical behavior as $k \rightarrow+\infty$.

In case of the Robin torsional rigidity, we prove:

Theorem 2. Let $r_{k}(\Omega, \beta)$ be defined by (2), with $\lambda(\Omega, \beta):=\tau^{-1}(\Omega, \beta)$. Then there holds

$$
\lim _{k \rightarrow+\infty} \frac{|\Omega|^{1 / 2}}{k^{3 / 2}} r_{k}(\Omega, \beta)=\beta h_{2}(H),
$$

where $h_{2}(H)$ denotes the 2-Cheeger constant of the unit area regular hexagon.

The notion of 2-Cheeger constant appearing in the statement of Theorem 2 is a variant of the classical definition (3) of Cheeger constant; precisely, the 2-Cheeger constant of a set $\Omega$ is given by

$$
h_{2}(\Omega):=\inf \left\{\frac{\operatorname{Per}\left(E, \mathbb{R}^{2}\right)}{|E|^{2}}: E \text { measurable, } E \subseteq \Omega\right\} .
$$

This generalization of Cheeger constant has already appeared in the literature, actually with the square of volume replaced by an arbitrary power with exponent $\alpha>1 / 2$, see [18, 19, 30].

In the same fashion as Theorem 1 is obtained by applying the analogous result proved in 8 ] for the Cheeger constant, combined with a tight control of the Robin eigenvalues in terms of the quotient perimeter over area, Theorem 2 is obtained by applying the analogous result for the 2-Cheeger constant, combined with a tight control of the Robin torsion in terms of the quotient perimeter over the square of the area. Actually, in order to prove Theorem 1, we need as a first step to settle a honeycomb-type result for the 2-Cheeger constant analogous to the one proved in [8] for the Cheeger constant. In turn, this requires to obtain a discrete Faber-Krahn inequality for the 2-Cheeger constant in the vein of [7] (but dealing just with convex polygons).

As a consequence of Theorems 1 and 2, we can also determine the asymptotic behaviour of similar problems where the energy is of supremal rather than additive type. Setting

$$
R_{k}(\Omega, \beta)=\left\{\begin{array}{l}
\inf \left\{\max _{i=1, \ldots, k} \lambda\left(E_{i}, \beta\right):\left\{E_{i}\right\} \in \mathcal{C}_{k}(\Omega)\right\} \text { if } \beta>0 \\
\sup \left\{\min _{i=1, \ldots, k} \lambda\left(E_{i}, \beta\right):\left\{E_{i}\right\} \in \mathcal{C}_{k}(\Omega)\right\} \text { if } \beta<0 .
\end{array}\right.
$$

we have:

Corollary 3. (i) If $R_{k}(\Omega, \beta)$ is defined by (5), with $\lambda(\Omega, \beta):=\lambda_{1}(\Omega, \beta)$, there holds

$$
\lim _{k \rightarrow+\infty} \frac{|\Omega|^{1 / 2}}{k^{1 / 2}} R_{k}(\Omega, \beta)=\beta h(H) ;
$$

(ii) If $R_{k}(\Omega, \beta)$ is defined by (5), with $\lambda(\Omega, \beta):=\tau^{-1}(\Omega, \beta)$, there holds

$$
\lim _{k \rightarrow+\infty} \frac{|\Omega|^{1 / 2}}{k^{1 / 2}} R_{k}(\Omega, \beta)=\beta h_{2}(H) .
$$

The detailed proofs of our results are presented hereafter with the following outline.

In Section 2, we provide some results about the 2-Cheeger constant which may have an autonomous interest, by showing by particular that it satisfies the honeycomb conjecture (with convex cells).

In Section 3, we establish some intermediate results towards the proofs of Theorems 1 and 2. which are crucial to make the connection between optimal Robin partitions and optimal 
Cheeger partitions: they consist essentially in settling good upper and lower bounds for $\lambda_{1}(\Omega, \beta)$ and $\tau(\Omega, \beta)$ in terms of geometrical quantities, holding under the assumption that the domain $\Omega$ is convex. Actually, in case of the Robin Laplacian eigenvalue and for $\beta>0$, an alternative simpler proof can be obtained by exploiting for the lower bound more rough inequalities not requiring convexity; nevertheless, since this direct approach is somehow related to the first eigenvalue and does not work for the Robin torsional rigidity, we preferred to follow the same guideline for both cases (see the final Remark 15 for more detailed comments in this direction).

Sections 4, 5, and 6 contain respectively the proofs of Theorems 1, Theorem 2 and Corollary 3.

\section{An Auxiliary Result about optimal 2-Cheeger Partitions}

The 2-Cheeger constant shares many features with the classical one. For instance, it is easy to check, by using the same arguments as for the classical Cheeger constant, that a 2Cheeger set $C_{2}(\Omega)$ (namely a solution to problem (4)) always exists; moreover a 2-Cheeger set is connected, its boundary is of class $\mathcal{C}^{1}$ and meets necessarily $\partial \Omega$ (and this occurs tangentially), and $\partial C_{2}(\Omega) \cap \Omega$ is made by arcs of circle (of radius $\left(2\left|C_{2}(\Omega)\right| h_{2}(\Omega) \mid\right)^{-1}$ ). We refer the interested reader to the proofs given in [30] for a similar notion of $\alpha$-Cheeger constant.

In this section we present some focused results about the 2-Cheeger constant, with the final scope of proving that it satisfies the honeycomb conjecture under convexity constraint on the cells, thus extending the result proved in 8 ] for the classical Cheeger constant.

We consider the following shape optimization problem, where $\mathcal{P}_{n}$ denotes the class of convex polygons with at most $n$ sides:

$$
\min \left\{|\Omega|^{3 / 2} h_{2}(\Omega): \Omega \in \mathcal{P}_{n}\right\} .
$$

Notice that, since the functional $\Omega \mapsto h_{2}(\Omega)$ is homogenoues of degree -3 under dilations, multiplying it by $|\Omega|^{3 / 2}$ we find a scale invariant functional. In particular, it turns out that problem (6) is well-posed. Moreover, in the next lemma we establish that the 2-Cheeger radius of an optimal polygon is uniquely determined through an explicit representation formula involving just three geometrical quantities: $|\partial \Omega|,|\Omega|$, and the functional $\Lambda(\Omega)$ defined by

$$
\Lambda(\Omega):=\sum_{i} \cot \left(\frac{\theta_{i}}{2}\right) .
$$

being $\theta_{1}, \ldots, \theta_{N}$ the inner angles of $\Omega$. The analogous result for the Cheeger constant can be found in [7, in a more general setting not requiring convexity.

Lemma 4. There exists a solution to problem (6), and any optimal polygon $\Omega$ admits a unique 2-Cheeger set $C_{2}(\Omega)$, which is determined by the equality

$$
\partial C_{2}(\Omega) \cap \Omega=\bigcup\left\{\Gamma_{\alpha}: \alpha \in \Theta(\Omega)\right\},
$$

where $\Theta$ denotes the family of inner angles of $\Omega$ and, for any $\alpha \in \Theta, \Gamma_{\alpha}$ is an arc of circle tangent to the two sides of $\partial \Omega$ forming the angle $\alpha$, of radius

$$
r_{2}(\Omega)=\frac{|\Omega|}{|\partial \Omega|+\sqrt{|\partial \Omega|^{2}-3|\Omega|(\Lambda(\Omega)-\pi)}} .
$$


Moreover, the 2-Cheeger constant of $\Omega$ is given by

$$
h_{2}(\Omega)=\frac{|\partial \Omega|-2 r_{2}(\Omega)(\Lambda(\Omega)-\pi)}{\left[|\Omega|-\left(r_{2}(\Omega)\right)^{2}(\Lambda(\Omega)-\pi)\right]^{2}} .
$$

Proof. The existence of an optimal polygon is straightforward: since we minimize over a closed subclass of the class of convex polygons a continuous and dilation invariant functionals, it is enough to apply the direct method of the Calculus of Variations working with the Hausdorff convergence.

In order to get the equality (8), assume that $\Omega \in \mathcal{P}_{N}$ is a solution to problem (6), and let $C_{2}(\Omega)$ be a 2 -Cheeger set of $\Omega$. Then it is readily seen that $C_{2}(\Omega)$ must touch every side of $\Omega$. (Namely, if by contradiction there exists a side which is not touched by $C_{2}(\Omega)$, we could construct a domain $\widetilde{\Omega}$, still belonging to $\mathcal{P}_{N}$, such that that $C_{2}(\Omega) \subset \widetilde{\Omega} \subset \Omega$. Then $|\widetilde{\Omega}|<|\Omega|$ and $h_{2}(\widetilde{\Omega})=h_{2}(\Omega)$, so that $|\Omega|^{3 / 2} h_{2}(\Omega)>|\widetilde{\Omega}|^{3 / 2} h_{2}(\widetilde{\Omega})$, against the optimality of $\Omega$ ).

As a consequence of the facts that $C_{2}(\Omega)$ meets every side of $\Omega$ and it is connected, we obtain that all the arcs of circle contained into $\partial C_{2}(\Omega) \cap \Omega$ must be tangent to two consecutive sides of $\Omega$.

Let us show that, for any $\alpha \in \Theta(\Omega)$, there exists an arc of type $\Gamma_{\alpha}$ such that $\Gamma_{\alpha} \subseteq$ $\partial C_{2}(\Omega) \cap \Omega$. Let $\alpha \in \Theta(\Omega)$ be fixed, and let $\Omega_{\alpha, r}$ be the domain obtained by "smoothing" the corner $\alpha$ by means of an arc of cirumference of radius $r$, tangent to the two sides of $\partial \Omega$ forming the angle $\alpha$. It is readily seen by geometric arguments that, for $r$ sufficiently small,

and

$$
\operatorname{Per}\left(\Omega_{\alpha, r}, \Omega\right)=|\partial \Omega|-2 r \cot \left(\frac{\alpha}{2}\right)+(\pi-\alpha) r
$$

Then,

$$
\left|\Omega_{\alpha, r}\right|=|\Omega|-r^{2} \cot \left(\frac{\alpha}{2}\right)+\left(\frac{\pi-\alpha}{2}\right) r^{2} .
$$

$$
\frac{\operatorname{Per}\left(\Omega_{\alpha, r}\right)}{\left|\Omega_{\alpha, r}\right|^{2}}=\frac{|\partial \Omega|-2 r\left[\tan \left(\frac{\pi-\alpha}{2}\right)-\left(\frac{\pi-\alpha}{2}\right)\right]}{\left\{|\Omega|-r^{2}\left[\tan \left(\frac{\pi-\alpha}{2}\right)-\left(\frac{\pi-\alpha}{2}\right)\right]\right\}^{2}} .
$$

Since the term in squared parenthesis is positive, we immediately see that the inequality $\frac{\operatorname{Per}\left(\Omega_{\alpha, r}\right)}{\left|\Omega_{\alpha, r}\right|^{2}}<\frac{|\partial \Omega|}{|\Omega|^{2}}$ is satisfied for $r$ sufficiently small.

In order to get the optimal radius, we have to minimize the function

$$
f(r):=\frac{|\partial \Omega|-2 r\left[\sum_{\alpha \in \Theta(\Omega)}\left[\tan \left(\frac{\pi-\alpha}{2}\right)\right]-\frac{\pi-\alpha}{2}\right]}{\left\{|\Omega|-r^{2}\left[\sum_{\alpha \in \Theta(\Omega)}\left[\tan \left(\frac{\pi-\alpha}{2}\right)\right]-\frac{\pi-\alpha}{2}\right]\right\}^{2}}=\frac{|\partial \Omega|-2 r(\Lambda(\Omega)-\pi)}{\left\{|\Omega|-r^{2}(\Lambda(\Omega)-\pi)\right\}^{2}} .
$$

In a neigbourhood of an optimal radius, this function is equal to or larger than the 2Cheeger constant of $\Omega$. In particular, we point out that $f(r)$ is strictly larger than $h_{2}(\Omega)$ if $r$ is above the critical value $\bar{r}$ for which two distinct arcs, each one tangent to two sides of $\partial \Omega$, lie on the same circumference (this can be easily seen by taking as a test in the definition of $h_{2}(\Omega)$ the intersection of the two sets obtained by smoothing two consecutive angles by an arc of circumference of radius $\bar{r}+\varepsilon$ : the quotient between perimeter and squared area of this test is strictly smaller than $f(\bar{r}+\varepsilon)$ ).

Therefore, we proceed to determine the critical points of $f$. 
By studying the first derivative $f^{\prime}(r)$, it is easy to see that $f$ is increasing in the interval $\left(r_{-}, r_{+}\right)$between the two roots of $f^{\prime}$,

$$
r_{ \pm}:=\frac{|\Omega|}{|\partial \Omega| \mp \sqrt{|\partial \Omega|^{2}-3|\Omega|(\Lambda(\Omega)-\pi)}},
$$

so that $f$ attains its minimum at $r_{-}$, which gives the value of the 2-Cheeger radius $r_{2}(\Omega)$. We have thus concluded the proof of (8)-(9), and (10) follows by definition.

Relying on Lemma 4, we obtain that the 2-Cheeger constant satisfies the following discrete Faber-Krahn inequality on convex polygons:

Proposition 5. The regular $n$-gon solves problem (6), with corresponding infimum equal to

$$
\gamma(n):=\frac{\left(2 \sqrt{n \tan \left(\frac{\pi}{n}\right)}+\sqrt{n \tan \left(\frac{\pi}{n}\right)+3 \pi}\right)^{3}}{8\left(n \tan \left(\frac{\pi}{n}\right)+\sqrt{n \tan \left(\frac{\pi}{n}\right)} \sqrt{n \tan \left(\frac{\pi}{n}\right)+3 \pi}+\pi\right)} .
$$

Proof. Thanks to the Lemma 4 , if $\Omega$ is an optimal polygon for problem (6), we can write the cost functional $h_{2}(\Omega)|\Omega|^{3 / 2}$ as a function depending only on the isoperimetric quotient $I(\Omega):=\frac{|\partial \Omega|}{|\Omega|^{1 / 2}}$ and on the constant $\Lambda(\Omega)$ introduced in (7). Indeed, starting from the equality (10), some straightforward computations give

$$
h_{2}(\Omega)|\Omega|^{3 / 2}=\Phi(I(\Omega), \Lambda(\Omega)),
$$

where

$$
\Phi(x, y):=\frac{x-\frac{2(y-\pi)}{\sqrt{x^{2}-3 y+3 \pi}+x}}{\left(1-\frac{y-\pi}{\left(\sqrt{x^{2}-3 y+3 \pi}+x\right)^{2}}\right)^{2}} .
$$

We observe that $\Lambda(\Omega)$ and $I(\Omega)$ obey the inequalities

$$
I^{2}(\Omega) \geq 4 \Lambda(\Omega) \geq 4 \Lambda\left(\Omega_{n}^{*}\right)=4 n \tan \left(\frac{\pi}{n}\right)(>4 \pi) .
$$

The former is the isoperimetric inequality for convex polygons (see for instance [17]), and becomes an equality when the polygon is circumscribed to a disk; the latter, recalling the definition of $\Lambda(\Omega)$, comes from the convexity of the map $t \mapsto \cot (t)$ on the interval $(0, \pi / 2)$, and becomes an equality whan all the inner angles of the polygon are equal.

We are thus led to minimize the function $\Phi(x, y)$ on the admissible region

$$
\mathcal{A}_{n}:=\left\{(x, y): x^{2} \geq 4 y \geq 4 n \tan \left(\frac{\pi}{n}\right)\right\} .
$$

We claim that the map $y \mapsto \Phi(x, y)$ is decreasing. Indeed, let us show that

$$
\frac{\partial \Phi(x, y)}{\partial y}=-\frac{x^{3}+\left(x^{2}+12 \pi-12 y\right) \sqrt{x^{2}-3 y+3 \pi}}{4\left(x^{2}-4 y+4 \pi\right)^{2}} \leq 0 \quad \text { if }(x, y) \in \mathcal{A}_{n},
$$

or equivalently that

$$
\Psi(x, y):=x^{3}+\left(x^{2}+12 \pi-12 y\right) \sqrt{x^{2}-3 y+3 \pi} \geq 0 \quad \text { if }(x, y) \in \mathcal{A}_{n} .
$$


Since

$$
\frac{\partial \Psi(x, y)}{\partial y}=-\frac{27\left(x^{2}-4 y+4 \pi\right)}{2 \sqrt{x^{2}-3 y+3 \pi}} \leq 0 \quad \text { if }(x, y) \in \mathcal{A}_{n}
$$

in order to obtain (13) it is enough to show that

$$
\eta(x):=\Psi\left(x, \frac{x^{2}}{4}\right)=x^{3}+\left(6 \pi-x^{2}\right) \sqrt{x^{2}+12 \pi} \geq 0 \quad \text { if } x \geq 2 \sqrt{n \tan \left(\frac{\pi}{n}\right)} .
$$

The latter inequality is readily checked, since the function $\eta$ turns out to be monotone decreasing, and satisfies the following asymptotic expansion as $x \rightarrow+\infty$ :

$$
\frac{\eta(x)}{x^{3}} \sim \frac{54 \pi^{2}}{x^{4}}+o\left(\frac{1}{x^{4}}\right) .
$$

We have thus proved (12), yielding

$$
\Phi(x, y) \geq \Phi\left(x, \frac{x^{2}}{4}\right)=\frac{\left(\sqrt{x^{2}+12 \pi}+2 x\right)^{3}}{16\left(x\left(\sqrt{x^{2}+12 \pi}+x\right)+4 \pi\right)}=: \zeta(x) .
$$

Next we observe that the map $x \mapsto \zeta(x)$ is increasing for $x \geq 2 \sqrt{n \tan \left(\frac{\pi}{n}\right)}$. Indeed, we have

$$
\zeta^{\prime}(x)=\frac{3\left(x\left(\sqrt{x^{2}+12 \pi}-x\right)+12 \pi\right)}{64 \pi} \geq 0 \quad \text { if } x \geq 2 \sqrt{\pi} .
$$

We conclude that the minimum of $\Phi(x, y)$ over the region $\mathcal{A}_{n}$ is attained at

$$
\left(x_{n}, y_{n}\right):=\left(2 \sqrt{n \tan \left(\frac{\pi}{n}\right)}, n \tan \left(\frac{\pi}{n}\right)\right),
$$

corresponding to the case when the convex polygon $\Omega$ is at the same time circumscribed to a disk and with all the inner angles equal, that is, $\Omega$ is the regular polygon. Accordingly, the expression of $\gamma(n)$ in (11) is found by evaluating $\Phi$ at $\left(x_{n}, y_{n}\right)$.

Finally, we arrive at the following honeycomb-type result, which extends to the case of the 2-Cheeger constant Corollary 9 in [8]:

Proposition 6. There holds

$$
\lim _{k \rightarrow+\infty} \frac{|\Omega|^{3 / 2}}{k^{5 / 2}} \inf \left\{\sum_{i=1}^{k} h_{2}\left(E_{i}\right):\left\{E_{i}\right\} \in \mathcal{C}_{k}(\Omega)\right\}=h_{2}(H),
$$

where $h_{2}(H)$ denotes the 2-Cheeger constant of the unit area regular hexagon.

Proof. The equality (14) follows by applying Theorem 2 in [8]. One has just check that assumptions (H1), (H2), (H3) therein are fulfilled. Assumptions (H1) and (H2) are satisfied, because the map $\Omega \mapsto h_{2}(\Omega)$ is monotone decreasing under inclusions and homogeneous of degree -3 under domain dilations. It remains to check assumptions (H3): according to Remark 4 (ii) in [8], in view of Proposition 5, it is enough to check that the map $n \mapsto \gamma(n)^{2 / 5}$ admits a decreasing and convex extension on $[2,+\infty)$. This can be done by elementary computations by exploiting the explicit expression of $\gamma(n)$ given in (11). 
Corollary 7. There holds

$$
\lim _{k \rightarrow+\infty} \frac{|\Omega|^{3 / 2}}{k^{5 / 2}} \inf \left\{\sum_{i=1}^{k} \frac{\left|\partial E_{i}\right|}{\left|E_{i}\right|^{2}}:\left\{E_{i}\right\} \in \mathcal{C}_{k}(\Omega)\right\}=h_{2}(H),
$$

where $h_{2}(H)$ denotes the 2-Cheeger constant of the unit area regular hexagon.

Proof. Set $m_{k}(\Omega)$ and $\widetilde{m}_{k}(\Omega)$ the infima at the r.h.s. of (15) and (14) respectively. The corollary follows straigthforward from Proposition 6 by noticing that $m_{k}(\Omega)=\widetilde{m}_{k}(\Omega)$. Indeed, let $\left\{E_{i}\right\} \in \mathcal{C}_{k}(\Omega)$. The inequality $h_{2}\left(E_{i}\right) \leq\left|\partial E_{i}\right| /\left|E_{i}\right|^{2}$ yields immediately $\widetilde{m}_{k}(\Omega) \leq$ $m_{k}(\Omega)$. Conversely, since $h_{2}\left(E_{i}\right)=\left|\partial C\left(E_{i}\right)\right| /\left|C\left(E_{i}\right)\right|^{2}$, there holds $\sum_{i} h_{2}\left(E_{i}\right) \geq m_{k}(\Omega)$, which yields $\widetilde{m}_{k}(\Omega) \geq m_{k}(\Omega)$.

\section{Some intermediate RESUlts}

We proceed separately in the cases of $\lambda_{1}(\Omega, \beta)$ and $\tau^{-1}(\Omega, \beta)$. The results of this section heavily rely on some works by Giorgi-Smits and Sperb in the former case, and by KeadyMcNabb in the latter case (see [20, 21, 27, 33]).

\subsection{Preliminaries to the proof of Theorem 1 ,}

Remark 8. It will be useful to keep in mind the following scaling law, which can be easily checked by change of variables:

$$
\lambda_{1}(t E, \beta)=\frac{1}{t^{2}} \lambda_{1}(E, t \beta) \quad \forall t>0 .
$$

Proposition 9. [upper and lower bounds for $\lambda_{1}(\Omega, \beta)$ ] Let $E$ be an open bounded Lipschitz set, and let $\mu_{2}(E)$ denote the first nonzero eigenvalue of the Neumann Laplacian in $E$. There holds:

$$
\begin{aligned}
& \lambda_{1}(E, \beta) \leq \beta \frac{|\partial E|}{|E|} \quad \forall \beta \in \mathbb{R} \backslash\{0\} \\
& \lambda_{1}(E, \beta) \geq \frac{1}{\frac{1}{\mu_{2}(E)}+\frac{|E|}{\beta|\partial E|}} \quad \forall \beta>0 \\
& \lim _{\beta \rightarrow 0} \frac{\lambda_{1}(E, \beta)}{\beta}=\frac{|\partial E|}{|E|} .
\end{aligned}
$$

Proof. The upper bound (17) is trivially obtained by taking as a test function $u \equiv 1$ in the definition of $\lambda_{1}(E, \beta)$. The lower bound (18) is due to Sperb, see [33. The asymptotic behaviour (19) is a direct consquence of (17)-(18) in case $\beta \rightarrow 0_{+}$but requires a further control from below in case $\beta \rightarrow 0_{-}$, see for instance [20, eq.(5)].

Proposition 10. [estimate of $\lambda_{1}(\Omega, \beta)$ by the width] Assume $\beta>0$. Let $E$ be an open bounded convex set, and let $w(E, \xi)$ denote the width of $E$ in some fixed direction $\xi$ (i.e., the distance between two support planes of $E$ orthogonal to $\xi)$. For every $\delta>0$, there exists a positive constant $C_{1}=C_{1}(\beta, \delta)$ such that

$$
w(E, \xi)<\delta \quad \Rightarrow \quad \lambda_{1}(E, \beta) \geq \frac{C_{1}}{w(E, \xi)} .
$$


Proof. Throughout the proof, we write for brevity $w$ in place of $w(E, \xi)$. We proceed in two steps. First we obtain the inequality

$$
\lambda_{1}(E, \beta) \geq \frac{1}{w^{2}} \lambda_{1}(I, w \beta)
$$

where $I=(-1 / 2,1 / 2)$ denotes the unit interval of the real line (and its Robin eigenvalue is meant in dimension 1) and then we show that the quotient

$$
\frac{\lambda_{1}(I, w \beta)}{w}
$$

admits a positive finite limit as $w \rightarrow 0^{+}$.

To prove (201), we slice $E$ in the direction $\xi$. Namely, we denote by $E_{\xi}$ the projection of $E$ onto the direction $\xi^{\perp}$, and for every $x \in E_{\xi}$ we set $\left(a_{x}, b_{x}\right):=E \cap(x+\mathbb{R} \xi)$. By Fubini's Theorem, if $u$ is a first eigenfunction for $\lambda_{1}(E, \beta)$, we have

$$
\begin{aligned}
\lambda_{1}(E, \beta) & =\frac{\int_{E}|\nabla u|^{2}+\beta \int_{\partial E} u^{2}}{\int_{E} u^{2}} \\
& \geq \frac{\int_{E_{\xi}} \int_{a_{x}}^{b_{x}}|\nabla u|^{2}(x, y) d y d x+\beta \int_{E_{\xi}}\left[u^{2}\left(a_{x}\right)+u^{2}\left(b_{x}\right)\right] d x}{\int_{E_{\xi}} \int_{a_{x}}^{b_{x}} u^{2}(x, y) d y d x} \\
& \geq \min _{x \in E_{\xi}} \frac{\int_{a_{x}}^{b_{x}}|\nabla u|^{2}(x, y) d y+\beta\left[u^{2}\left(a_{x}\right)+u^{2}\left(b_{x}\right)\right]}{\int_{a_{x}}^{b_{x}} u^{2}(x, y) d y} \\
& \geq \min _{x \in E_{\xi}} \lambda_{1}\left(\left(a_{x}, b_{x}\right), \beta\right) \geq \lambda_{1}\left(\left(-\frac{w}{2}, \frac{w}{2}\right), \beta\right)=\frac{1}{w^{2}} \lambda_{1}(I, w \beta) .
\end{aligned}
$$

where in the last line we have used the decreasing monotonicity of the map $B \mapsto \lambda_{1}(B, \beta)$ holding for balls $B$ for any $\beta>0$, and the scaling property (16).

To compute the limit of the quotient in (21), we solve the b.v.p. which defines $\lambda:=$ $\lambda_{1}(I, \alpha)$, that is we search for an even function on $I$ which satisfies

$$
\left\{\begin{array}{l}
-u^{\prime \prime}=\lambda u \quad \text { in } I \\
u^{\prime}\left(\frac{1}{2}\right)+\alpha u\left(\frac{1}{2}\right)=0 .
\end{array}\right.
$$

We have $u(x)=\cos (\sqrt{\lambda} x)$, and imposing the boundary condition we get the following relation between $\alpha$ and $\lambda$ :

$$
\frac{\alpha^{2}}{\lambda+\alpha^{2}}=\sin ^{2}\left(\frac{\sqrt{\lambda}}{2}\right) .
$$

In the limit as $\alpha \rightarrow 0$, we have $\lambda \rightarrow 0$; moreover, dividing (22) by $\lambda$ and passing to the limit as $\alpha \rightarrow 0$, we get

$$
\lim _{\alpha \rightarrow 0} \frac{\alpha^{2}}{\lambda\left(\lambda+\alpha^{2}\right)}=\frac{\sin ^{2}\left(\frac{\sqrt{\lambda}}{2}\right)}{\lambda}=\frac{1}{4} .
$$

We observe that $\alpha^{2}=o(\lambda)$; indeed, using (22), we see that

$$
\frac{4 \alpha^{2}}{\lambda} \sim \frac{\alpha^{2}}{\sin ^{2}\left(\frac{\sqrt{\lambda}}{2}\right)}=\lambda+\alpha^{2} \rightarrow 0 .
$$


Therefore, (23) can be rewritten as

$$
\lim _{\alpha \rightarrow 0} \frac{\alpha^{2}}{\lambda^{2}+o\left(\lambda^{2}\right)}=\frac{1}{4}
$$

yielding $\lim _{\alpha \rightarrow 0} \frac{\alpha}{\lambda}=\frac{1}{2}$. We conclude that

$$
\lim _{w \rightarrow 0} \frac{\lambda_{1}(I, w \beta)}{w}=2 \beta .
$$

\subsection{Preliminaries to the proof of Theorem $\mathbf{2}$,}

Remark 11. The Robin torsion satisfies a scaling law analogue to (16), which in this case reads:

$$
\tau^{-1}(t E, \beta)=\frac{1}{t^{4}} \tau^{-1}(E, t \beta) \quad \forall t>0 .
$$

Proposition 12. [upper and lower bounds for $\tau^{-1}(\Omega, \beta)$ ] Let $E$ be an open bounded Lipschits set with unit outer normal $\nu$, and let $\Sigma_{\infty}(E):=\int_{E} u_{\infty}$, being $u_{\infty}$ the unique solution to the boundary value problem

$$
\begin{cases}-\Delta u=1 & \text { in } E \\ u_{\nu}=-\frac{|E|}{|\partial E|} & \text { on } \partial E \\ \int_{\partial E} u=0 . & \end{cases}
$$

There holds:

$$
\begin{gathered}
\tau^{-1}(E, \beta) \leq \beta \frac{|\partial E|}{|E|^{2}} \quad \forall \beta \in \mathbb{R} \backslash\{0\} \\
\tau^{-1}(E, \beta) \geq \frac{1}{\Sigma_{\infty}(E)+\frac{|E|^{2}}{\beta|\partial E|}} \quad \forall \beta>0 \\
\lim _{\beta \rightarrow 0} \frac{\tau^{-1}(E, \beta)}{\beta}=\frac{|\partial E|}{|E|^{2}} .
\end{gathered}
$$

For the proof of Proposition 12 we need the following result; similar statements for the first Robin eigenvalue can be found in [1, Lemma 1] and [21, Lemma 2.2].

Lemma 13. Let $E$ be an open bounded Lipschitz domain.

(i) There exists a constant $M_{E}$ such that

$$
\int_{\partial E} u^{2} \leq \int_{E}|\nabla u|^{2}+M_{E}\left(\int_{E}|u|\right)^{2} \quad \forall u \in H^{1}(E) .
$$

(ii) One can find $\eta_{E}>0$ such that, for every $\eta \geq \eta_{E}$, there exists a constant $C(\eta)>0$, infinitesimal as $\eta \rightarrow+\infty$, such that

$$
\int_{\partial E} u^{2} \leq \eta \int_{E}|\nabla u|^{2}+\frac{|\partial E|}{|E|^{2}}(1+C(\eta))\left(\int_{E}|u|\right)^{2} \quad \forall u \in H^{1}(E) .
$$


Proof. (i) By Lemma 1 in [1, there exists a constant $C>0$ such that

$$
\int_{\partial E} u^{2} \leq \frac{1}{2} \int_{E}|\nabla u|^{2}+C \int_{E} u^{2} \quad \forall u \in H^{1}(E) .
$$

Then it is enough to show the following claim: for any given $C>0$, there exists $M>0$ sufficiently large such that

$$
C \int_{E} u^{2} \leq \frac{1}{2} \int_{E}|\nabla u|^{2}+M\left(\int_{E}|u|\right)^{2} \quad \forall u \in H^{1}(E) .
$$

The claim is readily cheked by contradiction. Assume there exists a sequence $M_{n} \rightarrow+\infty$ such that, for every $n \in \mathbb{N}$, there exists $u_{n} \in H^{1}(E)$, with $\int_{E} u_{n}^{2}=1$, satisfying

$$
C \geq \frac{1}{2} \int_{E}\left|\nabla u_{n}\right|^{2}+M_{n}\left(\int_{E}\left|u_{n}\right|\right)^{2} .
$$

Then the sequence $\left\{u_{n}\right\}$ turns out to be bounded in $H^{1}(E)$ so that, up to passing to a (not relabeled) subsequence, it converges to some function $u$ weaky in $H^{1}(E)$ and strongly both in $L^{1}(E)$ and $L^{2}(E)$. Recalling that $\int_{E} u_{n}^{2}=1$ for every $n$, we find $\int_{E} u^{2}=1$, whereas recalling that $M_{n} \rightarrow+\infty$, the inequality (29) implies $\int_{E}|u|=0$, contradiction.

(ii) We proceed by contradiction. If statement (ii) is false, we can find $\delta>0$ and a sequence $\left\{u_{n}\right\} \subset H^{1}(E)$ such that, for every $n \in \mathbb{N}$,

$$
\int_{\partial E} u_{n}^{2} \geq n \int_{E}\left|\nabla u_{n}\right|^{2}+\frac{|\partial E|}{|E|^{2}}(1+\delta)\left(\int_{E}\left|u_{n}\right|\right)^{2} .
$$

The above inequality implies in particular that $\int_{E}\left|\nabla u_{n}\right|^{2} \neq 0$ for every $n \in \mathbb{N}$ (otherwise $u_{n}$ is constant and we get a contradiction since $\delta>0$ ). Then we can define the functions $v_{n}:=\frac{u_{n}}{\left(\int_{E}\left|\nabla u_{n}\right|^{2}\right)^{1 / 2}}$, which satisfy

$$
\int_{\partial E} v_{n}^{2} \geq n+\frac{|\partial E|}{|E|^{2}}(1+\delta)\left(\int_{E}\left|v_{n}\right|\right)^{2} .
$$

On the other hand, from statement (i), and taking into account that $\int_{E}\left|\nabla v_{n}\right|^{2}=1$, we know that

$$
\int_{\partial E} v_{n}^{2} \leq 1+M_{E}\left(\int_{E}\left|v_{n}\right|\right)^{2} .
$$

Combining the two inequalities (30) and (31), we obtain a contradiction concluding the proof, provided we are able to show that the sequence $\int_{E}\left|v_{n}\right|$ remains bounded. Assume this is not the case. Then, the sequence $w_{n}:=\frac{v_{n}}{\int_{E}\left|v_{n}\right|}$ satisfies $\int_{E}\left|\nabla w_{n}\right|^{2} \rightarrow 0$. Since $\int_{E}\left|w_{n}\right|=1$, by exploiting claim (28) obtained above in the proof of statement (i), we infer that that $\left\{w_{n}\right\}$ remains bounded in $H^{1}(E)$. Hence, up to subsequences, it converges weakly in $H^{1}(E)$ and strongly in $L^{1}(E)$ to some limit $w$ which satisfies $\int_{E}|w|=1$ and $\int_{E}|\nabla w|^{2}=0$. It follows that $w$ is uniquely determined as $\frac{1}{|E|}$. By uniqueness of this limit, the whole sequence turns out converge strongly in $H^{1}(E)$ to $w$, and hence also strongly in $L^{2}(\partial E)$. Then (30) implies

$$
\frac{|\partial E|}{|E|^{2}}=\lim _{n} \int_{\partial E} w_{n}^{2} \geq \frac{|\partial E|}{|E|^{2}}(1+\delta),
$$

contradiction. 
Proof of Proposition 12, For the upper bound (25), it's enough to take $u \equiv 1$ in the definition of $\tau^{-1}(E, \beta)$. The lower bound (26) is due to Keady-McNabb, see [27, inequality (4.9)]. Let us prove the asymptotic behaviour (27). The limit as $\beta \rightarrow 0^{+}$is obtained immediately by combining the bounds (25) and (26). It remains to compute the limit as $\beta \rightarrow 0^{-}$. The inequality $\liminf _{\beta \rightarrow 0^{-}} \frac{\tau^{-1}(E, \beta)}{\beta} \geq \frac{|\partial E|}{|E|^{2}}$ follows immediately from (25). Let us show the converse inequality for the limsup. To that aim, we apply Lemma 13 by choosing $\eta=-\frac{1}{\beta}$ and taking as a function $u$ the solution to the Robin torsion problem, normalized so that $\int_{E}|u|=1$. We obtain

$\frac{\tau^{-1}(E, \beta)}{\beta}=\int_{\partial E} u^{2}+\frac{1}{\beta} \int_{E}|\nabla u|^{2} \leq \frac{|\partial E|}{|E|^{2}}\left(1+C\left(-\frac{1}{\beta}\right)\right)\left(\int_{E}|u|\right)^{2}=\frac{|\partial E|}{|E|^{2}}\left(1+C\left(-\frac{1}{\beta}\right)\right)$.

It follows that $\limsup _{\beta \rightarrow 0^{-}} \frac{\tau^{-1}(E, \beta)}{\beta} \leq \frac{|\partial E|}{|E|^{2}}$ as required.

Proposition 14. [estimate by the width] Assume $\beta>0$. Let $E$ be an open bounded convex set, and let $w(E, \xi)$ denote the width of $E$ in some fixed direction $\xi$ (i.e., the distance between two support planes of $E$ orthogonal to $\xi$ ). For every $\delta>0$, there exists a positive constant $C_{1}=C_{1}(\beta, \delta)$ such that

$$
w(E, \xi)<\delta \quad \Rightarrow \quad \tau^{-1}(E, \beta) \geq \frac{C_{1}}{w^{3}(E, \xi)} .
$$

Proof. We proceed in a similar way as in the proof of Proposition 10, We still set $w:=$ $w(E, \xi)$, and $I:=(-1 / 2,1 / 2)$, and we proceed again in two steps, showing first that

$$
\tau^{-1}(E, \beta) \geq \frac{1}{w^{4}\left|E_{\xi}\right|} \tau^{-1}(I, w \beta)
$$

and second that the quotient

$$
\frac{\tau^{-1}(I, w \beta)}{w}
$$

admits a positive finite limit as $w \rightarrow 0^{+}$.

To prove (32), we proceed by slicing. With the same notation as in the proof of Proposition 10. by using Fubini's Theorem and Hölder inequality, if $u$ is the solution to the Robin torsion problem, we have

$$
\begin{aligned}
\tau^{-1}(E, \beta) & =\frac{\int_{E}|\nabla u|^{2}+\beta \int_{\partial E} u^{2}}{\left(\int_{E}|u|\right)^{2}} \\
& \geq \frac{\int_{E_{\xi}} \int_{a_{x}}^{b_{x}}|\nabla u|^{2}(x, y) d y d x+\beta \int_{E_{\xi}}\left[u^{2}\left(a_{x}\right)+u^{2}\left(b_{x}\right)\right] d x}{\left(\int_{E_{\xi}} \int_{a_{x}}^{b_{x}}|u(x, y)| d y d x\right)^{2}} \\
& \geq \frac{1}{\left|E_{\xi}\right|} \min _{x \in E_{\xi}} \frac{\int_{a_{x}}^{b_{x}}|\nabla u|^{2}(x, y) d y+\beta\left[u^{2}\left(a_{x}\right)+u^{2}\left(b_{x}\right)\right]}{\left(\int_{a_{x}}^{b_{x}}|u(x, y)| d y\right)^{2}} \\
& \geq \frac{1}{\left|E_{\xi}\right|} \min _{x \in E_{\xi}} \tau^{-1}\left(\left(a_{x}, b_{x}\right), \beta\right) \geq \frac{1}{\left|E_{\xi}\right|} \tau^{-1}\left(\left(-\frac{w}{2}, \frac{w}{2}\right), \beta\right)=\frac{1}{w^{4}\left|E_{\xi}\right|} \lambda_{1}(I, w \beta) .
\end{aligned}
$$


where in the last line we have used the decreasing monotonicity of the map $B \mapsto \tau^{-1}(B, \beta)$ holding for balls $B$ for any $\beta>0$, and the scaling property (24).

To compute the limit of the quotient in (33), we solve the b.v.p. which defines $\tau^{-1}(I, \alpha)$, that is we search for an even function on $I$ which satisfies

$$
\left\{\begin{array}{l}
-u^{\prime \prime}=1 \quad \text { in } I \\
u^{\prime}\left(\frac{1}{2}\right)+\alpha u\left(\frac{1}{2}\right)=0 .
\end{array}\right.
$$

We find $u(x)=-\frac{x^{2}}{2}+\frac{1}{2 \alpha}+\frac{1}{8}$, with $\int_{I} u(x) d x=\frac{1}{12}+\frac{1}{2 \alpha}$.

We conclude that

$$
\lim _{w \rightarrow 0} \frac{\tau^{-1}(I, w \beta)}{w}=\lim _{w \rightarrow 0} \frac{1}{w} \frac{1}{\frac{1}{12}+\frac{1}{2 w \beta}}=2 \beta .
$$

\section{Proof of Theorem 1}

We start from the following fact: setting

$$
m_{k}(\Omega)=\inf \left\{\sum_{i=1}^{k} \frac{\left|\partial E_{i}\right|}{\left|E_{i}\right|}:\left\{E_{i}\right\} \in \mathcal{C}_{k}(\Omega)\right\}
$$

there holds

$$
\lim _{k \rightarrow+\infty} \frac{|\Omega|^{1 / 2}}{k^{3 / 2}} m_{k}(\Omega)=h(H)
$$

This is obtained immediately by applying Corollary 9 in [8], and arguing as in the proof of Corollary 7 .

We now proceed separately in the two cases $\beta>0$ and $\beta<0$, assuming without loss of generality that $|\Omega|=1$.

- Case $\beta>0$.

In view of (17) in Proposition 9 and (35), we have

$$
\limsup _{k \rightarrow+\infty} \frac{r_{k}(\Omega, \beta)}{k^{3 / 2}} \leq \limsup _{k \rightarrow+\infty} \beta \frac{m_{k}(\Omega)}{k^{3 / 2}}=\beta h(H)
$$

We are going to show that

$$
\liminf _{k \rightarrow+\infty} \frac{r_{k}(\Omega, \beta)}{k^{3 / 2}} \geq \liminf _{k \rightarrow+\infty} \beta \frac{m_{k}(\Omega)}{k^{3 / 2}}=\beta h(H)
$$

By (36) , we can choose $\bar{k}$ sufficiently large so that, for every $k \geq \bar{k}$, it holds

$$
\frac{r_{k}(\Omega, \beta)}{k^{3 / 2}} \leq 2 \beta h(H) \text {. }
$$

Then, for every $k \geq \bar{k}$, we let $\left\{\omega_{1}^{*}, \ldots, \omega_{k}^{*}\right\}$ be a convex cluster in $\Omega$ such that

$$
\sum_{i=1}^{k} \lambda_{1}\left(\omega_{i}^{*}, \beta\right) \leq r_{k}(\Omega, \beta)+1
$$


For a given $\varepsilon>0$, we introduce the class of convex bodies such that the ratio between the width in a direction orthogonal to a diameter and the diameter is at least $\varepsilon$. We denote it by

$$
\operatorname{Conv}(\varepsilon):=\left\{E \in \mathcal{K}^{2}: \frac{w(E, \xi)}{\operatorname{diam}(E)} \geq \varepsilon \text { for some } \xi \in S^{1} \text { orthogonal to a diameter }\right\} .
$$

Then we consider the following families of indices associated with the clusters $\left\{\omega_{1}^{*}, \ldots, \omega_{k}^{*}\right\}$

$$
\theta_{k, \varepsilon}:=\left\{i \in\{1, \ldots, k\}: \omega_{i}^{*} \in \operatorname{Conv}(\varepsilon)\right\}, \quad \theta_{k, \varepsilon}^{c}:=\{1, \ldots, k\} \backslash \theta_{\varepsilon} .
$$

We can estimate $r_{k}(\Omega ; \beta)$ from below as follows

$$
1+r_{k}(\Omega, \beta) \geq \sum_{i=1}^{k} \lambda_{1}\left(\omega_{i}^{*}, \beta\right) \geq \sum_{i \in \theta_{k, \varepsilon}} \lambda_{1}\left(\omega_{i}^{*}, \beta\right) .
$$

We are thus led to introduce the auxiliary problems

$$
r_{k, \varepsilon}(\Omega, \beta):=\inf \left\{\sum_{i \in \theta_{k, \varepsilon}} \lambda_{1}\left(E_{i}, \beta\right):\left\{E_{i}\right\} \in \mathcal{C}_{\sharp \theta_{k, \varepsilon}}(\Omega), E_{i} \in \operatorname{Conv}(\varepsilon)\right\} .
$$

In order to show (37), we are going to exploit the lower bound (40), and to estimate from below $r_{k, \varepsilon}(\Omega, \beta)$ in terms of the corresponding auxiliary problems

$$
m_{k, \varepsilon}(\Omega):=\inf \left\{\sum_{i \in \theta_{k, \varepsilon}} \frac{\left|\partial E_{i}\right|}{\left|E_{i}\right|}:\left\{E_{i}\right\} \in \mathcal{C}_{\sharp \theta_{k, \varepsilon}}(\Omega), E_{i} \in \operatorname{Conv}(\varepsilon)\right\} .
$$

We divide the remaining of the proof in three steps.

Step I: for $k$ large enough, it holds

$$
\sharp \theta_{k, \varepsilon} \geq\left(1-C \varepsilon^{1 / 3}\right) k,
$$

where $C$ stands for a positive constant, not depending on $k$ nor on $\varepsilon$. Consequently,

$$
\lim _{k \rightarrow+\infty} \frac{m_{k, \varepsilon}(\Omega)}{\left(\sharp \theta_{k, \varepsilon}\right)^{3 / 2}}=h(H) .
$$

Let us first observe that (44) is a straightforward consequence of (43). Indeed, by definition it is clear that $m_{k, \varepsilon}(\Omega) \geq m_{k}(\Omega)$ On the other hand, provided $\varepsilon<1 / 2$, we have $H \in$ $\operatorname{Conv}(\varepsilon)$, so that a configuration which is asymptotically optimal, in the limit as $k \rightarrow+\infty$, for $m_{k}(\Omega)$ is admissible for $m_{k, \varepsilon}(\Omega)$. This yields (44) since, by (43), we know that $\sharp \theta_{k, \varepsilon} \sim k$ as $k \rightarrow+\infty$.

In order to estimate the cardinality of $\theta_{k, \varepsilon}$, we first obtain a bound on the width of the cells $\omega_{i}^{*}$ and $\Omega_{i}^{*}$. Hereafter we denote for brevity $d_{i}$ and $w_{i}$ the diameter of such cells, and their width in the direction orthogonal to a diameter.

We have

$$
1=|\Omega| \geq \sum_{i=1}^{k}\left|\omega_{i}^{*}\right| \geq \frac{1}{2} \sum_{i=1}^{k} d_{i} w_{i} \geq \frac{1}{2} \sum_{i \in \theta_{\varepsilon}^{c}} \frac{w_{i}^{2}}{\varepsilon}
$$

where we have used the fact that, by convexity, the area of each cell is bounded from below by $\left(d_{i} w_{i}\right) / 2$ and the fact that, by definition, for cells in $\theta_{k, \varepsilon}^{c}$, it holds $d_{i} \geq w_{i} / \varepsilon$. Now we must proceed to estimate the cardinality of $\theta_{k, \varepsilon}$.

Starting from (45) and using Proposition 10, the elementary inequality between the 2-mean and the (-1)-mean, inequality (39), and inequality (38), we obtain, for $k$ large enough: 


$$
\begin{aligned}
1 & \geq \frac{1}{2 \varepsilon} \sum_{i \in \theta_{k, \varepsilon}^{c}} w_{i}^{2} \geq \frac{C_{1}^{2}}{2 \varepsilon} \sum_{i \in \theta_{k, \varepsilon}^{c}}\left(\frac{1}{\lambda_{1}\left(\omega_{i}^{*}, \beta\right)}\right)^{2} \\
& \geq \frac{C_{1}^{2}}{2 \varepsilon} \frac{\left(\sharp \theta_{k, \varepsilon}^{c}\right)^{3}}{\left(\sum_{i \in \theta_{k, \varepsilon}^{c}} \lambda_{1}\left(\omega_{i}^{*}, \beta\right)\right)^{2}} \geq \frac{C_{1}^{2}}{2 \varepsilon} \frac{\left(\sharp \theta_{k, \varepsilon}^{c}\right)^{3}}{\left(3 \beta h(H) k^{3 / 2}\right)^{2}} .
\end{aligned}
$$

Hence inequality in (43) is satisfied, for $k$ large enough, with $C=\left(\frac{18 \beta^{2} h^{2}(H)}{C_{1}^{2}}\right)^{1 / 3}$.

Step II: For $k$ large enough, the infima $r_{k, \varepsilon}(\Omega, \beta)$ and $m_{k, \varepsilon}(\Omega, \beta)$ introduced in (41) and (42) satisfy

$$
\liminf _{k \rightarrow+\infty} \frac{r_{k, \varepsilon}(\Omega, \beta)}{k^{3 / 2}} \geq(1-\varepsilon) \beta \liminf _{k \rightarrow+\infty} \frac{m_{k, \varepsilon}(\Omega)}{k^{3 / 2}}
$$

Let $\left\{E_{i}\right\}$ be a cluster in $\mathcal{C}_{\sharp \theta_{k, \varepsilon}}(\Omega)$, with $E_{i} \in \operatorname{Conv}(\varepsilon)$. In order to estimate $\lambda_{1}\left(E_{i}, \beta\right)$, we introduce the following constant depending only on $\varepsilon$ :

$$
K_{\varepsilon}:=\inf \left\{\mu_{2}(E)|E|: E \in \operatorname{Conv}(\varepsilon)\right\} .
$$

Notice that $K_{\varepsilon}$ is strictly positive (and attained) because the family $\operatorname{Conv}(\varepsilon)$ is closed in the Hausdorff topology. Then we distinguish the cells of the cluster $\left\{E_{i}\right\}$ into two disjoint subclasses, in which we are able to provide respectively a lower and an upper bound for $\lambda_{1}\left(E_{i}, \beta\right)$.

Class 1 : cells with $\beta\left|\partial E_{i}\right| \leq \varepsilon K_{\varepsilon}$. For such cells, it holds

$$
\lambda_{1}\left(E_{i}, \beta\right) \geq(1-\varepsilon) \beta \frac{\left|\partial E_{i}\right|}{\left|E_{i}\right|} .
$$

Namely, using the lower bound (18) given by Proposition 9, we have

$$
\lambda_{1}\left(E_{i}, \beta\right) \geq \frac{1}{\frac{1}{\mu_{2}\left(E_{i}\right)}+\frac{\left|E_{i}\right|}{\beta\left|\partial E_{i}\right|}}=\beta \frac{\left|\partial E_{i}\right|}{\left|E_{i}\right|}\left(1-\frac{\beta\left|\partial E_{i}\right|}{\beta\left|\partial E_{i}\right|+\mu_{2}\left(E_{i}\right)\left|E_{i}\right|}\right) .
$$

Therefore, the required estimate (47) is satisfied provided

$$
\frac{\beta\left|\partial E_{i}\right|}{\beta\left|\partial E_{i}\right|+\mu_{2}\left(E_{i}\right)\left|E_{i}\right|} \leq \varepsilon,
$$

which holds for cells of Class 1 , as the inequality $\beta\left|\partial E_{i}\right| \leq \varepsilon K_{\varepsilon} \leq \varepsilon \mu_{2}\left(E_{i}\right)\left|E_{i}\right|$ is in force.

Class 2 : cells with $\beta\left|\partial E_{i}\right|>\varepsilon K_{\varepsilon}$. For such cells, it holds

$$
\lambda_{1}\left(E_{i}, \beta\right) \leq \beta \frac{\left|\partial E_{i}\right|}{\left|E_{i}\right|} \leq \frac{32 \beta^{2}}{\varepsilon^{2} K_{\varepsilon}} .
$$

Namely, using the upper bound (17) given by Proposition 9, and the elementary estimates $\left|\partial E_{i}\right| \leq 4 d_{i},\left|E_{i}\right| \geq \frac{1}{2} d_{i} w_{i}$ (being $d_{i}$ the diameter of $E_{i}$, and $w_{i}$ its width in a direction orthogonal to a diameter), we obtain

$$
\lambda_{1}\left(E_{i}, \beta\right) \leq \beta \frac{\left|\partial E_{i}\right|}{\left|E_{i}\right|} \leq \beta \frac{4 d_{i}}{\frac{1}{2} d_{i} w_{i}}=\frac{8 \beta}{w_{i}}
$$


Therefore, the required estimate (48) is satisfied provided

$$
w_{i} \geq \frac{\varepsilon^{2} K_{\varepsilon}}{4 \beta}
$$

which holds for cells of Class 2 . Indeed for such cells the inequality $4 d_{i} \beta \geq \varepsilon K_{\varepsilon}$ is in force, which yields $d_{i} \geq \frac{\varepsilon K_{\varepsilon}}{4 \beta}$. In turn, the latter inequality implies (49) since $E_{i} \in \operatorname{Conv}(\varepsilon)$.

Now we proceed to prove the estimate in (46). Let $\left\{E_{i}\right\}$ be a cluster in $\mathcal{C}_{\sharp \theta_{k, \varepsilon}}(\Omega)$, with $E_{i} \in \operatorname{Conv}(\varepsilon)$. We set for brevity

$$
\begin{aligned}
& \theta_{k, \varepsilon}^{(1)}\left(\left\{E_{i}\right\}\right):=\left\{i \in \theta_{k, \varepsilon}: E_{i} \text { is of Class } 1\right\} \\
& \theta_{k, \varepsilon}^{(2)}\left(\left\{E_{i}\right\}\right):=\left\{i \in \theta_{k, \varepsilon}: E_{i} \text { is of Class } 2\right\} .
\end{aligned}
$$

We start by noticing that, in the limit as $k \rightarrow+\infty$, the infimum which defines $m_{k, \varepsilon}(\Omega)$ has the same asymptotic behaviour if we restrict the the sum to indices in the family $\theta_{k, \varepsilon}^{(1)}\left(\left\{E_{i}\right\}\right)$. More precisely, we have:

$$
\begin{aligned}
& \liminf _{k \rightarrow+\infty} \frac{1}{k^{3 / 2}} \inf \left\{\sum_{i \in \theta_{k, \varepsilon}} \frac{\left|\partial E_{i}\right|}{\left|E_{i}\right|}:\left\{E_{i}\right\} \in \mathcal{C}_{\sharp \theta_{k, \varepsilon}}(\Omega), E_{i} \in \operatorname{Conv}(\varepsilon)\right\}= \\
& \liminf _{k \rightarrow+\infty} \frac{1}{k^{3 / 2}} \inf \left\{\sum_{i \in \theta_{k, \varepsilon}^{(1)}\left(\left\{E_{i}\right\}\right)} \frac{\left|\partial E_{i}\right|}{\left|E_{i}\right|}:\left\{E_{i}\right\} \in \mathcal{C}_{\sharp \theta_{k, \varepsilon}}(\Omega), E_{i} \in \operatorname{Conv}(\varepsilon)\right\} .
\end{aligned}
$$

Indeed, on one hand we know from (43) and (44) that

$$
m_{k, \varepsilon}(\Omega)=\inf \left\{\sum_{i \in \theta_{k, \varepsilon}} \frac{\left|\partial E_{i}\right|}{\left|E_{i}\right|}:\left\{E_{i}\right\} \in \mathcal{C}_{\sharp \theta_{k, \varepsilon}}(\Omega), E_{i} \in \operatorname{Conv}(\varepsilon)\right\} \sim h(H) k^{3 / 2} ;
$$

on the other hand, for any admissible cluster $\left\{E_{i}\right\}$ in $\mathcal{C}_{\sharp \theta_{k, \varepsilon}}(\Omega)$, with $E_{i} \in \operatorname{Conv}(\varepsilon)$, by (48) we have

$$
\sum_{i \in \theta_{k, \varepsilon}^{(2)}\left(\left\{E_{i}\right\}\right)} \frac{\left|\partial E_{i}\right|}{\left|E_{i}\right|} \leq \frac{32 \beta}{\varepsilon^{2} K_{\varepsilon}} k
$$

where the quantity $\frac{32 \beta}{\varepsilon^{2} K_{\varepsilon}}$ is independent of $k$. Then (150) follows by (51) and (52). 
Now, exploiting (47) and (50), we obtain

$$
\begin{aligned}
\liminf _{k \rightarrow+\infty} \frac{r_{k, \varepsilon}(\Omega, \beta)}{k^{3 / 2}} & =\liminf _{k \rightarrow+\infty} \frac{1}{k^{3 / 2}} \inf \left\{\sum_{i \in \theta_{k, \varepsilon}} \lambda_{1}\left(E_{i}, \beta\right):\left\{E_{i}\right\} \in \mathcal{C}_{\sharp \theta_{k, \varepsilon}}(\Omega), E_{i} \in \operatorname{Conv}(\varepsilon)\right\} \\
& \geq \liminf _{k \rightarrow+\infty} \frac{1}{k^{3 / 2}} \inf \left\{\sum_{i \in \theta_{k, \varepsilon}^{(1)}\left(\left\{E_{i}\right\}\right)} \lambda_{1}\left(E_{i}, \beta\right):\left\{E_{i}\right\} \in \mathcal{C}_{\sharp \theta_{k, \varepsilon}}(\Omega), E_{i} \in \operatorname{Conv}(\varepsilon)\right\} \\
& \geq(1-\varepsilon) \beta \liminf _{k \rightarrow+\infty} \frac{1}{k^{3 / 2}} \inf \left\{\sum_{i \in \theta_{k, \varepsilon}^{(1)}\left(\left\{E_{i}\right\}\right)} \frac{\left|\partial E_{i}\right|}{\left|E_{i}\right|}:\left\{E_{i}\right\} \in \mathcal{C}_{\sharp \theta_{k, \varepsilon}}(\Omega), E_{i} \in \operatorname{Conv}(\varepsilon)\right\} \\
& =(1-\varepsilon) \beta \liminf _{k \rightarrow+\infty} \frac{1}{k^{3 / 2}} \inf \left\{\sum_{i \in \theta_{k, \varepsilon}} \frac{\left|\partial E_{i}\right|}{\left|E_{i}\right|}:\left\{E_{i}\right\} \in \mathcal{C}_{\sharp \theta_{k, \varepsilon}}(\Omega), E_{i} \in \operatorname{Conv}(\varepsilon)\right\} \\
& =(1-\varepsilon) \beta \liminf _{k \rightarrow+\infty} \frac{m_{k, \varepsilon}(\Omega)}{k^{3 / 2}} .
\end{aligned}
$$

Step III: The lower bound (37) holds true.

By (40), (43), and (46), we have

$$
\begin{aligned}
\liminf _{k \rightarrow+\infty} \frac{1+r_{k}(\Omega, \beta)}{k^{3 / 2}} & =\liminf _{k \rightarrow+\infty} \frac{r_{k, \varepsilon}(\Omega, \beta)}{k^{3 / 2}} \geq(1-\varepsilon) \beta \liminf _{k \rightarrow+\infty} \frac{m_{k, \varepsilon}(\Omega)}{k^{3 / 2}} \\
& =(1-\varepsilon) \beta \liminf _{k \rightarrow+\infty}\left(\frac{\sharp \theta_{k, \varepsilon}}{k}\right)^{3 / 2} \frac{m_{k, \varepsilon}(\Omega)}{\left(\sharp \theta_{k, \varepsilon}\right)^{3 / 2}} \\
& \geq(1-\varepsilon) \beta\left(1-C \varepsilon^{1 / 3}\right)^{3 / 2} \liminf _{k \rightarrow+\infty} \frac{m_{k, \varepsilon}(\Omega)}{\left(\sharp \theta_{k, \varepsilon}\right)^{3 / 2}} .
\end{aligned}
$$

By (53) and (44), we conclude that

$$
\liminf _{k \rightarrow+\infty} \frac{r_{k}(\Omega, \beta)}{k^{3 / 2}} \geq(1-\varepsilon) \beta\left(1-C \varepsilon^{1 / 3}\right)^{3 / 2} h(H) .
$$

Eventually, in the limit as $\varepsilon \rightarrow 0^{+}$, we obtain (37).

- Case $\beta<0$.

In view of inequality (17) given by Proposition 9 and the asymptotic equality (35), and taking into account that $\beta<0$, we have

$$
\begin{aligned}
\limsup _{k \rightarrow+\infty} \frac{r_{k}(\Omega, \beta)}{k^{3 / 2}} & =\limsup _{k \rightarrow+\infty} \frac{1}{k^{3 / 2}} \sup \left\{\sum_{i=1}^{k} \lambda_{1}\left(E_{i}, \beta\right):\left\{E_{i}\right\} \in \mathcal{C}_{k}(\Omega)\right\} \\
& \leq \limsup _{k \rightarrow+\infty} \frac{1}{k^{3 / 2}} \sup \left\{\sum_{i=1}^{k} \beta \frac{\left|\partial E_{i}\right|}{\left|E_{i}\right|}:\left\{E_{i}\right\} \in \mathcal{C}_{k}(\Omega)\right\} \\
& =\limsup _{k \rightarrow+\infty} \frac{\beta}{k^{3 / 2}} \inf \left\{\sum_{i=1}^{k} \frac{\left|\partial E_{i}\right|}{\left|E_{i}\right|}:\left\{E_{i}\right\} \in \mathcal{C}_{k}(\Omega)\right\} \\
& =\limsup _{k \rightarrow+\infty} \frac{\beta m_{k}(\Omega)}{k^{3 / 2}}=\beta h(H) .
\end{aligned}
$$


To prove the converse estimate, since $|\Omega|=1$, we observe that for every $\varepsilon>0$ there exists $k_{\varepsilon}$ such that for every $k \geq k_{\varepsilon}$, the set $\Omega$ contains the convex $k$-cluster

$$
\left\{\left(\frac{1-\varepsilon}{k}\right)^{\frac{1}{2}} C(H), \ldots,\left(\frac{1-\varepsilon}{k}\right)^{\frac{1}{2}} C(H)\right\}
$$

given by $k$ copies of the Cheeger set $C(H)$ of the unit area regular hexagon, each one scaled so to have area $\frac{1-\varepsilon}{k}$. Hence, using also the asymptotic behaviour (19) and the scaling property (16), we get

$$
\begin{aligned}
\liminf _{k \rightarrow+\infty} \frac{r_{k}(\Omega, \beta)}{k^{3 / 2}} & =\liminf _{k \rightarrow+\infty} \frac{1}{k^{3 / 2}} \sup \left\{\sum_{i=1}^{k} \lambda_{1}\left(E_{i}, \beta\right):\left\{E_{i}\right\} \in \mathcal{C}_{k}(\Omega)\right\} \\
& \geq \liminf _{k \rightarrow+\infty} \frac{1}{k^{3 / 2}} k \lambda_{1}\left(\left(\frac{1-\varepsilon}{k}\right)^{\frac{1}{2}} C(H), \beta\right) \\
& =\liminf _{k \rightarrow+\infty} \frac{1}{k^{3 / 2}} \frac{k^{2}}{(1-\varepsilon)} \lambda_{1}\left(C(H), \frac{(1-\varepsilon)^{1 / 2} \beta}{k^{1 / 2}}\right) \\
& =\liminf _{k \rightarrow+\infty} \frac{1}{k^{3 / 2}} \frac{k^{2}}{(1-\varepsilon)} \frac{(1-\varepsilon)^{1 / 2} \beta}{k^{1 / 2}} \frac{|\partial C(H)|}{|C(H)|}=\frac{1}{(1-\varepsilon)^{1 / 2}} \beta h(H) .
\end{aligned}
$$

Since $\varepsilon>0$ is arbitrary, the proof is concluded.

\section{Proof of Theorem 2}

On the basis of the results established in Section [3.2, the proof of Theorem 2 proceeds along the same line as the proof of Theorem 1. Hence we present it more concisely, often referring to the proof of Theorem 1 whenever the two proofs are basically the same. We still assume that $|\Omega|=1$, and we now set

$$
m_{k}(\Omega)=\inf \left\{\sum_{i=1}^{k} \frac{\left|\partial E_{i}\right|}{\left|E_{i}\right|^{2}}:\left\{E_{i}\right\} \in \mathcal{C}_{k}(\Omega)\right\}
$$

- Case $\beta>0$.

In view of inequality (25) in Proposition 12 and Corollary 17, we have

$$
\limsup _{k \rightarrow+\infty} \frac{r_{k}(\Omega, \beta)}{k^{5 / 2}} \leq \limsup _{k \rightarrow+\infty} \beta \frac{m_{k}(\Omega)}{k^{5 / 2}}=\beta h_{2}(H)
$$

We have to prove that

$$
\liminf _{k \rightarrow+\infty} \frac{r_{k}(\Omega, \beta)}{k^{5 / 2}} \geq \liminf _{k \rightarrow+\infty} \beta \frac{m_{k}(\Omega)}{k^{5 / 2}}=\beta h_{2}(H) .
$$

By (56), we can choose $\bar{k}$ sufficiently large so that, for every $k \geq \bar{k}$, it holds

$$
\frac{r_{k}(\Omega, \beta)}{k^{5 / 2}} \leq 2 \beta h_{2}(H) .
$$

Then, for every $k \geq \bar{k}$, we let $\left\{\omega_{1}^{*}, \ldots, \omega_{k}^{*}\right\}$ be a convex cluster in $\Omega$ such that

$$
\sum_{i=1}^{k} \tau^{-1}\left(\omega_{i}^{*}, \beta\right) \leq r_{k}(\Omega, \beta)+1 .
$$


For a given $\varepsilon>0$, we introduce the class of convex bodies $\operatorname{Conv}(\varepsilon)$ defined as in the proof of Theorem 1, and accordingly we consider the families of indices $\theta_{k, \varepsilon}$ associated with the cluster $\left\{\omega_{1}^{*}, \ldots, \omega_{k}^{*}\right\}$ as done in such proof.

Also in the present setting, we have the lower bound

$$
1+r_{k}(\Omega, \beta) \geq \sum_{i=1}^{k} \tau^{-1}\left(\omega_{i}^{*}, \beta\right) \geq \sum_{i \in \theta_{k, \varepsilon}} \tau^{-1}\left(\omega_{i}^{*}, \beta\right) .
$$

Hence we introduce the auxiliary problems:

$$
\begin{gathered}
r_{k, \varepsilon}(\Omega, \beta):=\inf \left\{\sum_{i \in \theta_{k, \varepsilon}} \tau^{-1}\left(E_{i}, \beta\right):\left\{E_{i}\right\} \in \mathcal{C}_{\sharp \theta_{k, \varepsilon}}(\Omega), E_{i} \in \operatorname{Conv}(\varepsilon)\right\} \\
m_{k, \varepsilon}(\Omega):=\inf \left\{\sum_{i \in \theta_{k, \varepsilon}} \frac{\left|\partial E_{i}\right|}{\left|E_{i}\right|^{2}}:\left\{E_{i}\right\} \in \mathcal{C}_{\sharp \theta_{k, \varepsilon}}(\Omega), E_{i} \in \operatorname{Conv}(\varepsilon)\right\} .
\end{gathered}
$$

We divide the remaining of the proof in three steps.

Step I: for $k$ large enough, it holds

$$
\sharp \theta_{k, \varepsilon} \geq\left(1-C \varepsilon^{3 / 5}\right) k,
$$

where $C$ stands for a positive constant, not depending on $k$ nor on $\varepsilon$. Consequently,

$$
\lim _{k \rightarrow+\infty} \frac{m_{k, \varepsilon}(\Omega)}{\left(\sharp \theta_{k, \varepsilon}\right)^{3 / 2}}=h(H) .
$$

The equality (64) is deduced from (63) exactly in the same way as in the proof of Theorem 1. We proceed to prove the estimate (63). Denoting by $d_{i}$ and $w_{i}$ the diameter of the cells $\omega_{i}^{*}$ or $\Omega_{i}^{*}$, and their width in the direction orthogonal to a diameter, we still have the inequality obtained in (45), namely

$$
1 \geq \frac{1}{2} \sum_{i \in \theta_{\varepsilon}^{c}} \frac{w_{i}^{2}}{\varepsilon}
$$

- Estimate of $\sharp \theta_{k, \varepsilon}$. Starting from (65) and using Proposition 14, the elementary inequality between the $\left(\frac{2}{3}\right)$-mean and the (-1)-mean, inequality (58), and inequality (59), we obtain, for $k$ large enough:

$$
\begin{aligned}
1 & \geq \frac{1}{2 \varepsilon} \sum_{i \in \theta_{k, \varepsilon}^{c}} w_{i}^{2} \geq \frac{C_{1}^{2 / 3}}{2 \varepsilon} \sum_{i \in \theta_{k, \varepsilon}^{c}} \tau^{2 / 3}\left(\omega_{i}^{*}, \beta\right) \\
& \geq \frac{C_{1}^{2 / 3}}{2 \varepsilon} \frac{\left(\sharp \theta_{k, \varepsilon}^{c}\right)^{5 / 3}}{\left(\sum_{i \in \theta_{k, \varepsilon}^{c}} \tau^{-1}\left(\omega_{i}^{*}, \beta\right)\right)^{2 / 3}} \geq \frac{C_{1}^{2 / 3}}{2 \varepsilon} \frac{\left(\sharp \theta_{k, \varepsilon}^{c}\right)^{5 / 3}}{\left(3 \beta h_{2}(H) k^{5 / 2}\right)^{2 / 3}} .
\end{aligned}
$$

Hence inequality (63) is satisfied, for $k$ large enough, with $C=\left(\frac{72 \beta^{2} h_{2}^{2}(H)}{C_{1}^{2}}\right)^{1 / 5}$. 
Step II: For $k$ large enough, the infima $r_{k, \varepsilon}(\Omega, \beta)$, and $m_{k, \varepsilon}(\Omega, \beta)$, introduced in (61) -(62) satisfy

$$
\liminf _{k \rightarrow+\infty} \frac{r_{k, \varepsilon}(\Omega, \beta)}{k^{3 / 2}} \geq(1-\varepsilon) \beta \liminf _{k \rightarrow+\infty} \frac{m_{k, \varepsilon}(\Omega)}{k^{3 / 2}} .
$$

Let $\left\{E_{i}\right\}$ be a cluster in $\mathcal{C}_{\sharp \theta_{k, \varepsilon}}(\Omega)$, with $E_{i} \in \operatorname{Conv}(\varepsilon)$. In order to estimate $\tau^{-1}\left(E_{i}, \beta\right)$, we introduce the following constant depending only on $\varepsilon$ :

$$
K_{\varepsilon}:=\inf \left\{\left(\Sigma_{\infty}(E)\right)^{-1}|E|^{2}: E \in \operatorname{Conv}(\varepsilon)\right\},
$$

the constant $\Sigma_{\infty}(E)$ being defined as in Proposition [12, We observe that $K_{\varepsilon}$ is strictly positive and attained because the family $\operatorname{Conv}(\varepsilon)$ is closed in the Hausdorff topology, and the functional $\left(\Sigma_{\infty}(E)\right)^{-1}|E|^{2}$ is continuous and scale-invariant (indeed, $\Sigma_{\infty}$ is easily seen from its definition to be homogeneous of degree 4 under dilations). Then we distinguish the cells of the cluster $\left\{E_{i}\right\}$ into the same two subclasses as in the proof of Theorem 1 ,

Class 1 : cells with $\beta\left|\partial E_{i}\right| \leq \varepsilon K_{\varepsilon}$. For such cells, it holds

$$
\tau^{-1}\left(E_{i}, \beta\right) \geq(1-\varepsilon) \beta \frac{\left|\partial E_{i}\right|}{\left|E_{i}\right|^{2}} .
$$

Namely, using the lower bound (26) given by Proposition 12, we have

$$
\tau^{-1}\left(E_{i}, \beta\right) \geq \frac{1}{\Sigma_{\infty}\left(E_{i}\right)+\frac{\left|E_{i}\right|^{2}}{\beta\left|\partial E_{i}\right|}}=\beta \frac{\left|\partial E_{i}\right|}{\left|E_{i}\right|^{2}}\left(1-\frac{\Sigma_{\infty}\left(E_{i}\right)}{\Sigma_{\infty}\left(E_{i}\right)+\frac{\left|E_{i}\right|^{2}}{\beta\left|\partial E_{i}\right|}}\right) .
$$

Therefore, the required estimate (67) is satisfied provided

$$
\frac{\Sigma_{\infty}\left(E_{i}\right)}{\Sigma_{\infty}\left(E_{i}\right)+\frac{\left|E_{i}\right|^{2}}{\beta\left|\partial E_{i}\right|}} \leq \varepsilon,
$$

which holds since cells of Class 1 satisfy $\beta\left|\partial E_{i}\right| \leq \varepsilon K_{\varepsilon} \leq \varepsilon\left(\Sigma_{\infty}\left(E_{i}\right)\right)^{-1}\left|E_{i}\right|^{2}$.

Class 2 : cells with $\beta\left|\partial E_{i}\right|>\varepsilon K_{\varepsilon}$. For such cells, it holds

$$
\tau^{-1}\left(E_{i}, \beta\right) \leq \beta \frac{\left|\partial E_{i}\right|}{\left|E_{i}\right|^{2}} \leq \frac{4^{5} \beta^{4}}{\varepsilon^{5} K_{\varepsilon}^{3}} .
$$

Namely, using the upper bound (25) given by Proposition 12, the elementary estimates $\left|\partial E_{i}\right| \leq 4 d_{i},\left|E_{i}\right| \geq \frac{1}{2} d_{i} w_{i}$, and the fact that we are dealing with cells of Class 2 in $\operatorname{Conv}(\varepsilon)$, which satisfy in particular $4 d_{i} \beta \geq \varepsilon K_{\varepsilon}$, we get

$$
\tau^{-1}\left(E_{i}, \beta\right) \leq \beta \frac{\left|\partial E_{i}\right|}{\left|E_{i}\right|^{2}} \leq \beta \frac{4 d_{i}}{\frac{1}{4} d_{i}^{2} w_{i}^{2}}=\frac{16 \beta}{d_{i} w_{i}^{2}} \leq \frac{16 \beta}{\varepsilon^{2} d_{i}^{3}} \leq \frac{16 \beta}{\varepsilon^{2}\left(\frac{\varepsilon K_{\varepsilon}}{4 \beta}\right)^{3}}=\frac{4^{5} \beta^{4}}{\varepsilon^{5} K_{\varepsilon}^{3}} .
$$

Now, having at our disposal the bounds (67) and (68) for cells of Class 1 and Class 2 respectively, the estimate in (66) can be proved in the analogous way as in Theorem 1 . The idea is that the infimum which defines $m_{k, \varepsilon}(\Omega)$ has the same asymptotic behaviour if we restrict the the sum to indices $i \in \theta_{k, \varepsilon}\left(\left\{E_{i}\right\}\right)$ such that $E_{i}$ is of Class 1 . It is enough to follow the proof of (46), with the obvious modifications in the scaling factors, and exploiting (67)-(68) in place of (47)-(48).

Step III: The lower bound (57) holds true. On can repeat the same arguments used for Step III in the proof of Theorem 1, with the obvious modifications (in particular, we exploit (60), (63), (66), and (64)). 
- Case $\beta<0$. We address the reader to the proof of Theorem 1 in case $\beta<0$, which runs exactly in the same way after suitably modifying the scaling factors.

\section{Proof of Corollary 3}

We give the proof only in case (i), as case (ii) is completely analogous.

- Case $\beta>0$. From the definition of $R_{k}(\Omega, \beta)$, we have $k R_{k}(\Omega, \beta) \geq r_{k}(\Omega, \beta)$, so that

$$
\limsup _{k \rightarrow+\infty} \frac{|\Omega|^{1 / 2}}{k^{1 / 2}} R_{k}(\Omega, \beta) \geq \limsup _{k \rightarrow+\infty} \frac{|\Omega|^{1 / 2}}{k^{3 / 2}} r_{k}(\Omega, \beta)=\beta h(H),
$$

where in the last equality we have applied Theorem 1 .

To prove the converse inequality, assume $|\Omega|=1$, and observe that for every $\varepsilon>0$ there exists $k_{\varepsilon}$ such that for every $k \geq k_{\varepsilon}$, the set $\Omega$ contains the convex $k$-cluster

$$
\left\{\left(\frac{1-\varepsilon}{k}\right)^{\frac{1}{2}} C(H), \ldots,\left(\frac{1-\varepsilon}{k}\right)^{\frac{1}{2}} C(H)\right\}
$$

given by $k$ copies of the Cheeger set $C(H)$ of the unit area regular hexagon, each one scaled so to have area $\frac{1-\varepsilon}{k}$. We get

$$
\begin{aligned}
\liminf _{k \rightarrow+\infty} \frac{R_{k}(\Omega, \beta)}{k^{1 / 2}} & \leq \liminf _{k \rightarrow+\infty} \frac{1}{k^{1 / 2}} \lambda_{1}\left(\left(\frac{1-\varepsilon}{k}\right)^{\frac{1}{2}} C(H), \beta\right) \\
& =\liminf _{k \rightarrow+\infty} \frac{1}{k^{1 / 2}} \frac{k}{1-\varepsilon} \lambda_{1}\left(C(H), \frac{\beta(1-\varepsilon)^{1 / 2}}{k^{1 / 2}}\right) \\
& =\liminf _{k \rightarrow+\infty} \frac{1}{k^{1 / 2}} k \frac{\beta}{k^{1 / 2}} \frac{1}{(1-\varepsilon)^{1 / 2}} \frac{|\partial C(H)|}{|C(H)|}=\frac{1}{(1-\varepsilon)^{1 / 2}} \beta h(H) .
\end{aligned}
$$

The parameter $\varepsilon>0$ being arbitrary, we conclude the proof.

- Case $\beta<0$. From the definition of $R_{k}(\Omega, \beta)$, we have in this case $k R_{k}(\Omega, \beta) \leq r_{k}(\Omega, \beta)$, so that

$$
\liminf _{k \rightarrow+\infty} \frac{|\Omega|^{1 / 2}}{k^{1 / 2}} R_{k}(\Omega, \beta) \leq \liminf _{k \rightarrow+\infty} \frac{|\Omega|^{1 / 2}}{k^{3 / 2}} r_{k}(\Omega, \beta)=\beta h(H),
$$

where in the last equality we have applied Theorem 1.

To prove the converse inequality, we proceed as above. We assume without loss of generality that $|\Omega|=1$, and we exploit the fact that asymptotically $\Omega$ contains the convex $k$-cluster $\left\{\left(\frac{1-\varepsilon}{k}\right)^{\frac{1}{2}} C(H), \ldots,\left(\frac{1-\varepsilon}{k}\right)^{\frac{1}{2}} C(H)\right\}$ to obtain

$$
\liminf _{k \rightarrow+\infty} \frac{R_{k}(\Omega, \beta)}{k^{1 / 2}} \geq \liminf _{k \rightarrow+\infty} \frac{1}{k^{1 / 2}} k \lambda_{1}\left(\frac{C(H)}{k^{1 / 2}}, \beta\right)=\beta h(H) .
$$

Remark 15. For $\beta>0$, a more direct proof of Theorem 1 (and consequently of Corollary 3 (i)) can be performed by using the following lower bound in place of (18):

$$
\lambda_{1}(\Omega, \beta) \geq \beta h(\Omega)-\beta^{2} .
$$

We point out that this inequality, which must be attributed to Bossel [5], holds true without any assumption on $\Omega$. It is in general quite rough (for instance, the right hand side can have negative sign for some $\Omega$ and $\beta$ ), but it becomes useful as soon as the Cheeger constant $h(\Omega)$ becomes large, which is typically the case in a partition with a large number 
of cells. For the sake of completeness, we enclose a short independent proof. Recall that the functional formulation of the Cheeger constant reads

$$
h(\Omega)=\inf _{v \in B V\left(\mathbb{R}^{2}\right) \backslash\{0\}, v=0 \text { on } \mathbb{R}^{2} \backslash \Omega} \frac{|D v|\left(\mathbb{R}^{2}\right)}{\int_{\Omega}|v|} .
$$

Then, letting $u$ be a first Robin eigenfunction, extended to 0 out of $\Omega$, and taking $v=u^{2}$ in the minimization problem (171), gives

$$
\begin{aligned}
\beta h(\Omega) & \leq \frac{\beta \int_{\Omega}\left|\nabla\left(u^{2}\right)\right|+\beta \int_{\partial \Omega} u^{2}}{\int_{\Omega} u^{2}}=\frac{\beta \int_{\Omega} 2|u \nabla u|+\beta \int_{\partial \Omega} u^{2}}{\int_{\Omega} u^{2}} \\
& \leq \frac{\int_{\Omega}|\nabla u|^{2}+\beta^{2} \int_{\Omega} u^{2}+\beta \int_{\partial \Omega} u^{2}}{\int_{\Omega} u^{2}}=\lambda_{1}(\Omega, \beta)+\beta^{2},
\end{aligned}
$$

which is exactly (70). Now, by exploiting such estimate, it is immediate to obtain the required inequality (37) in the proof of Theorem 1.

We emphasize that this more direct approach does not work for the Robin torsional ridigity, which still requires the finer inequality on convex sets. Indeed, Bossel's approach seem to be unadaptable to the case of torsional rigidity; as well, by arguing as above one arrives at the lower bound

$$
\tau^{-1}(\Omega, \beta) \geq \frac{\beta}{|\Omega|} h(\Omega)-\frac{\beta^{2}}{|\Omega|},
$$

which does not allow obtain the required inequality (57) in the proof of Theorem 2. Instead, (72) may be used to prove the statements analougue to Theorem 2 and Corollary 3 (ii) for the more unusual functional $\lambda(\Omega, \beta):=|\Omega| \tau^{-1}(\Omega, \beta)$.

\section{REFERENCES}

[1] G. A. Afrouzi and K. J. Brown, On principal eigenvalues for boundary value problems with indefinite weight and Robin boundary conditions, Proc. Amer. Math. Soc. 127 (1999), no. 1, 125-130.

[2] C. Bandle and A. Wagner, Domain perturbations for elliptic problems with Robin boundary conditions of opposite sign, Algebra i Analiz, translation in St. Petersburg Math. J. 28 (2017), no. 2, 153-170.

[3] B. Bogosel and B. Velichkov, A multiphase shape optimization problem for eigenvalues: qualitative study and numerical results, SIAM J. Numer. Anal. 54 (2016), no. 1, 210-241.

[4] V. Bonnaillie-Noël, B. Helffer, and G. Vial, Numerical simulations for nodal domains and spectral minimal partitions, ESAIM Control Optim. Calc. Var. 16 (2010), no. 1, 221-246.

[5] M. Bossel, Membranes élastiquement liées: extension du théorème de Rayleigh-Faber-Krahn et de l'inégalité de Cheeger, C. R. Acad. Sci. Paris Sér. I Math. 302 (1986), no. 1, 47-50.

[6] D. Bucur, G. Buttazzo, and A. Henrot, Existence results for some optimal partition problems, Adv. Math. Sci. Appl. 8 (1998), no. 2, 571-579.

[7] D. Bucur and I. Fragalà, A Faber-Krahn Inequality for the Cheeger Constant of N-gons, J. Geom. Anal. 26 (2016), no. 1, 88-117.

[8] D. Bucur, I. Fragalà, B. Velichkov, and G. Verzini, On the honeycomb conjecture for a class of minimal convex partitions, Arxiv Preprint, arXiv:1703.05383 (2017).

[9] D. Bucur and A. Giacomini, Faber-Krahn inequalities for the Robin-Laplacian: a free discontinuity approach, Arch. Ration. Mech. Anal. 218 (2015), no. 2, 757-824. MR 3375539

[10] D. Bucur and A. Giacomini, Shape optimization problems with Robin conditions on the free boundary, Ann. Inst. H. Poincaré Anal. Non Linéaire 33 (2016), no. 6, 1539-1568. MR 3569242

[11] L. A. Caffarelli and D. Kriventsov, A free boundary problem related to thermal insulation, Comm. Partial Differential Equations 41 (2016), no. 7, 1149-1182. MR 3528530

[12] L. A. Caffarelli and F. H. Lin, An optimal partition problem for eigenvalues, J. Sci. Comput. 31 (2007), no. $1-2,5-18$. 
[13] M. Caroccia, Cheeger N-clusters, Calc. Var. Partial Differential Equations 56 (2017), no. 2, 56:30. MR 3610172

[14] M. Caroccia and F. Maggi, A sharp quantitative version of Hales' isoperimetric honeycomb theorem, J. Math. Pures Appl. (9) 106 (2016), no. 5, 935-956.

[15] M. Conti, S. Terracini, and G. Verzini, An optimal partition problem related to nonlinear eigenvalues, J. Funct. Anal. 198 (2003), no. 1, 160-196.

[16] M. Conti, S. Terracini, and G. Verzini, On a class of optimal partition problems related to the Fučík spectrum and to the monotonicity formulae, Calc. Var. Partial Differential Equations 22 (2005), no. 1, $45-72$.

[17] G. Crasta, I. Fragalà, and F. Gazzola, A sharp upper bound for the torsional rigidity of rods by means of web functions, Arch. Ration. Mech. Anal. 164 (2002), no. 3, 189-211.

[18] A. Figalli, F. Maggi, and A. Pratelli, A note on Cheeger sets, Proc. Amer. Math. Soc. 137 (2009), no. $6,2057-2062$.

[19] N. Fusco, F. Maggi, and A. Pratelli, Stability estimates for certain Faber-Krahn, isocapacitary and Cheeger inequalities, Ann. Sc. Norm. Super. Pisa Cl. Sci. (5) 8 (2009), no. 1, 51-71.

[20] T. Giorgi and R. Smits, Monotonicity results for the principal eigenvalue of the generalized Robin problem, Illinois J. Math. 49 (2005), no. 4, 1133-1143.

[21] T. Giorgi and R. Smits, Eigenvalue estimates and critical temperature in zero fields for enhanced surface superconductivity, Z. Angew. Math. Phys. 58 (2007), no. 2, 224-245.

[22] T. C. Hales, The honeycomb conjecture, Discrete Comput. Geom. 25 (2001), no. 1, 1-22.

[23] B. Helffer, Domaines nodaux et partitions spectrales minimales (d'après B. Helffer, T. HoffmannOstenhof et S. Terracini), Séminaire: Équations aux Dérivées Partielles. 2006-2007, Sémin. Équ. Dériv. Partielles, École Polytech., Palaiseau, 2007, pp. Exp. No. VIII, 23.

[24] B. Helffer, On spectral minimal partitions: a survey, Milan J. Math. 78 (2010), no. 2, 575-590.

[25] B. Helffer, T. Hoffmann-Ostenhof, and S. Terracini, Nodal domains and spectral minimal partitions, Ann. Inst. H. Poincaré Anal. Non Linéaire 26 (2009), no. 1, 101-138.

[26] A. Henrot, Extremum problems for eigenvalues of elliptic operators, Frontiers in Mathematics, Birkhäuser Verlag, Basel, 2006.

[27] G. Keady and A. McNabb, Functions with constant Laplacian satisfying homogeneous Robin boundary conditions, IMA J. Appl. Math. 50 (1993), no. 3, 205-224.

[28] G. P. Leonardi, An overview on the Cheeger problem, Pratelli, A., Leugering, G. (eds.) New trends in shape optimization., International Series of Numerical Mathematics, Springer (Switzerland), vol. 166, 2016, pp. 117-139.

[29] E. Parini, An introduction to the Cheeger problem, Surv. Math. Appl. 6 (2011), 9-21.

[30] A. Pratelli and G. Saracco, On the generalized Cheeger problem and an application to 2d strips, Rev. Mat. Iberoam. 33 (2017), no. 1, 219-237.

[31] M. Ramos, H. Tavares, and S. Terracini, Extremality conditions and regularity of solutions to optimal partition problems involving Laplacian eigenvalues, Arch. Ration. Mech. Anal. 220 (2016).

[32] A.Y. Solynin and V. A. Zalgaller, An isoperimetric inequality for logarithmic capacity of polygons, Ann. of Math. (2) 159 (2004), no. 1, 277-303.

[33] R.P. Sperb, Untere und obere Schranken für den tiefsten Eigenwert der elastisch gestützten Membran, Z. Angew. Math. Phys. 23 (1972), 231-244.

(Dorin Bucur) Laboratoire de Mathématiques UMR 5127, Université de Savoie, Campus ScienTIFIQUe, 73376 LE-Bourget-Du-LaC (France)

E-mail address: dorin.bucur@univ-savoie.fr

(Ilaria Fragalà) Dipartimento di Matematica, Politecnico di Milano, Piazza Leonardo da Vinci, 32, 20133 Milano (Italy)

E-mail address: ilaria.fragala@polimi.it 